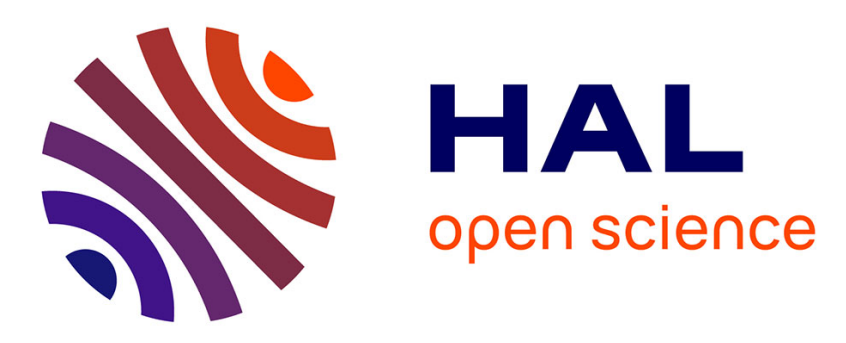

\title{
On lack-of-knowledge theory in structural mechanics
} François Louf, Paul Enjalbert, Pierre Ladevèze, Thierry Romeuf

\section{To cite this version:}

François Louf, Paul Enjalbert, Pierre Ladevèze, Thierry Romeuf. On lack-of-knowledge theory in structural mechanics. Comptes Rendus Mécanique, 2010, 338 (7-8), pp.424-433. 10.1016/j.crme.2010.07.012 . hal-01648324

\section{HAL Id: hal-01648324 https://hal.science/hal-01648324}

Submitted on 18 Dec 2019

HAL is a multi-disciplinary open access archive for the deposit and dissemination of scientific research documents, whether they are published or not. The documents may come from teaching and research institutions in France or abroad, or from public or private research centers.
L'archive ouverte pluridisciplinaire HAL, est destinée au dépôt et à la diffusion de documents scientifiques de niveau recherche, publiés ou non, émanant des établissements d'enseignement et de recherche français ou étrangers, des laboratoires publics ou privés. 


\title{
On lack-of-knowledge theory in structural mechanics
}

\section{Sur la théorie des méconnaissances en mécanique des structures}

\author{
François Louf ${ }^{\mathrm{a}, *}$, Paul Enjalbert ${ }^{\mathrm{a}}$, Pierre Ladevèze ${ }^{\mathrm{a}, 1}$, Thierry Romeuf ${ }^{\mathrm{b}}$ \\ a LMT-Cachan (ENS Cachan/CNRS/UPMC/PRES UniverSud Paris), 61, avenue du Président Wilson, 94235 Cachan cedex, France \\ ${ }^{\mathrm{b}}$ EADS Astrium, route de Verneuil BP96, 78133 Les Mureaux cedex, France
}

Today, the validation of complex structural models - i.e. the assessment of their quality compared to an experimental reference - remains a major issue. Strictly speaking, the validation problem consists in comparing the response of the numerical model (whether deterministic or stochastic) with complete reality. A first answer to this problem, using Lack-Of-Knowledge (LOK) theory, was introduced at LMT-Cachan. This theory is an attempt to "model the unknown" by taking all the sources of uncertainties, including modeling errors, into account through the concept of basic LOKs. In this article, we introduce basic LOKs associated with both the amplitudes and directions of excitations. These basic LOKs are propagated rigorously throughout the mechanical model in order to determine intervals (with stochastic bounds) within which lies a given quantity of interest (stress or displacement). Then, we introduce a strategy for the reduction of lack of knowledge, which we illustrate through an academic example.

La validation de modèles structuraux complexes - c'est-à-dire la vérification de leur qualité vis-à-vis d'une référence expérimentale - demeure un verrou scientifique fort. Le véritable problème de validation consiste à comparer la réponse du modèle numérique, qu'il soit déterministe ou pas, avec la réponse de toutes les structures réelles, dans tous les environnements possible. Un premier élément de réponse à ce problème a été introduit via la théorie des méconnaissances au LMT-Cachan. Afin de "modéliser l'inconnu», cette théorie prend en compte toutes les incertitudes, en incluant les erreurs de modèles, à travers le concept de méconnaissances de base. Dans le cet article, on introduit des méconnaissances de base sur les excitations (amplitude et direction). Ces méconnaissances de base sont ensuite propagées à travers le modèle mécanique afin de déterminer des intervalles dont les bornes sont probabilistes, contenant une quantité d'intérêt (contrainte ou déplacement). Ensuite une stratégie de réduction des méconnaissances de base par apport d'information expérimentale est présentée sur un exemple académique.

\footnotetext{
* Corresponding author.

E-mail addresses: francois.louf@Imt.ens-cachan.fr (F. Louf), paul.enjalbert@lmt.ens-cachan.fr (P. Enjalbert), pierre.ladeveze@lmt.ens-cachan.fr (P. Ladevèze), thierry.romeuf@space.eads.net (T. Romeuf).

1 EADS Foundation Chair, Advanced Computational Structural Mechanics.
} 


\section{Introduction}

Model validation is becoming quite a popular topic in structural mechanics, even though some people say that a model can be only invalidated, but never validated. Much has been written on this controversial issue (see [1-5]). On the positive side, the term "validation" is very often used by engineers to designate a procedure, defined through practical rules and experiments, which ensures that the structure being designed will perform as expected after it is built.

Many methods have been developed for the validation of structural models using experimental information. In particular, indirect or parametric methods lead to updated mass, stiffness and damping matrices through changes in the physical parameters of the model. The associated cost functions can be classified into three categories: input residuals [6,7], output residuals [8,9], and the particular residual called the "Constitutive Relation Error (CRE)" [10]. The CRE was also applied to stochastic model updating in [11].

However, even after the model has been updated, there may remain some uncertainties concerning the structural parameters.

The challenge is to clear up these uncertainties through the development of practical tools which can be applied to actual engineering problems. In this article, we introduce Lack-Of-Knowledge (LOK) theory, whose objective is to "model the unknown" conservatively.

The concept of lack of knowledge, initially introduced in [12] and [13] for stiffness-related sources of error, consists in globalizing the various sources of errors on the substructure's level using scalar internal variables (called LOK variables) defined over intervals whose upper and lower bounds follow probabilistic laws. The pair of stochastic bounds associated with each substructure is called a basic LOK. From these basic LOKs, one can calculate the effective LOK of a quantity of interest (e.g. an eigenfrequency, a displacement or a stress) over the whole structure, leading to an interval with stochastic bounds. This is achieved by propagating the basic LOKs and the associated probabilistic laws rigorously throughout the mechanical model.

In Section 2, we briefly present the basic LOKs associated with sources of error concerning the stiffness. Then, we expand the LOK theory in order to take into account, for quasi-static problems, uncertainties concerning the amplitudes and directions of the applied forces. Finally, we present the approach leading to the effective LOK of a quantity of interest.

In Section 3, the proposed LOK theory is illustrated through an academic example. A cantilever beam is excited at its free end by a force whose transverse position and direction are uncertain. In order to represent these uncertainties, we introduce two kinds of basic LOKs: one associated with the direction of the applied force, and the other associated with the amplitude of the uncertain twisting torque generated by the transverse position of the excitation. By propagating a first set of basic LOKs, we obtain the envelope of the model's response for two quantities of interest.

Then, in order to validate the LOK model thus defined, we undertake to compare the response envelope with experimental reality. In order to do that, we use simulated experimental data obtained from a finite element model in which the random positions and amplitudes of the applied force were modeled by Gaussian laws. A Monte Carlo simulation led to $99 \%$-probability bounds of the two quantities of interest.

A first comparison of the responses from the experimental and LOK models shows that the basic LOKs chosen were too large. In order to update the basic LOKs, we introduce a cost function measuring the difference between the two response envelopes. By minimizing this cost function, we obtained an optimum set of basic LOKs leading to the perfect agreement of the two response envelopes.

\section{Model validation: the real problem}

At the heart of any approach to the actual validation problem in the context of engineering reality is the description of parameters such as material uncertainties. Probabilistic modeling has become increasingly popular [14]. There are also other approaches which do not involve probability laws, such as in [15-18].

Here, we present an approach, called Lack-Of-Knowledge (LOK) theory, which attempts to give a pragmatic answer to the problem of model validation in the context of engineering reality. This could be viewed as an extension of the concept of safety coefficients [12,19].

\subsection{The concept of basic LOKs}

Let us consider a family of quasi-identical real structures subjected to a given (but not very well-known) environment. For the sake of simplicity, we are focusing on static problems. From here on, random variables are denoted $X(\theta)$, where $\theta$ belongs to the space of random events.

\subsubsection{Basic LOKs of the stiffness}

The structure is modeled as an assembly of substructures $E(E \in \mathbb{E})$ whose connections can be viewed as special substructures. The corresponding scale is assumed to be consistent with the outputs of interest being sought. We also assume that an elastic, deterministic finite element model is sufficient to predict the response of the "structure" in the usual sense. 
The starting point of the LOK theory consists in associating with each substructure $E$ a pair of scalar internal state variables $\left(m_{\mathrm{E}}^{-}, m_{\mathrm{E}}^{+}\right)$, called the "basic LOKs", such that:

$$
-m_{\mathrm{E}}^{-}(\theta) \overline{\mathbb{K}}_{\mathrm{E}} \leqslant \mathbb{K}_{\mathrm{E}}(\theta)-\overline{\mathbb{K}}_{\mathrm{E}} \leqslant m_{\mathrm{E}}^{+}(\theta) \overline{\mathbb{K}}_{\mathrm{E}}
$$

where $\overline{\mathbb{K}}_{\mathrm{E}}$ is the stiffness matrix of the deterministic reference model and $\mathbb{K}_{\mathrm{E}}(\theta)$ is the stiffness matrix of a real structure belonging to the family being studied.

In this formal expression, the inequalities must be considered to hold for the eigenvalues. The basic LOKs $\left(m_{\mathrm{E}}^{+}\right.$and $m_{\mathrm{E}}^{-}$) can be set-valued, but usually follow given probability laws. Let us note that a simpler description would consist in replacing the interval $\left[-m_{\mathrm{E}}^{-}(\theta) ; m_{\mathrm{E}}^{+}(\theta)\right]$ by a fuzzy interval depending on only one stochastic variable.

Introducing:

- $\bar{e}_{E}(\underline{U})$, the elastic energy of substructure $E \in \mathbb{E}$ calculated with the displacement field $\underline{U}$ of the deterministic reference model,

- and $e_{E}(\underline{U}, \theta)$, the elastic energy of substructure $E \in \mathbb{E}$ calculated with the displacement field $\underline{U}$ of a real structure,

we get:

$$
\left(1-m_{\mathrm{E}}^{-}(\theta)\right) \bar{e}_{E}(\underline{U}) \leqslant e_{E}(\underline{U}, \theta) \leqslant\left(1+m_{\mathrm{E}}^{+}(\theta)\right) \bar{e}_{E}(\underline{U})
$$

2.1.2. Basic LOKs of the applied forces

The definition of the basic LOKs of the applied forces (and, more generally, on the description of the environment) is similar to that of the basic LOKs about the structural stiffness. Let us consider a force distribution $\underline{F}$ which can be written as:

$$
\underline{F}=\sum_{E \in \mathbb{E}} \underline{F}_{E}
$$

where $\underline{F}_{E}$ is the restriction of $\underline{F}$ to substructure $E$ at the boundary of $E$ or over the $E$-domain. Furthermore, let us consider that:

$$
\underline{F}_{E}=\lambda_{E} \underline{Z}_{E}
$$

where $\lambda_{E}$ is the amplitude and $\underline{Z}_{E}$ is the direction of the load restriction $\underline{F}_{E}$, normalized as follows:

$$
\underline{Z}_{E}^{T} \underline{Z}_{E}=1
$$

In this case, the associated basic LOK is a triplet for each $E \in \mathbb{E}$. This triplet is generally stochastic, but can be set-valued: we introduce two scalar LOKs for the amplitude of the force and one scalar LOK for its direction. This LOK operator $\underline{m}_{F}=$ $\left(m_{\lambda_{E}}^{-}(\theta), m_{\lambda_{E}}^{+}(\theta), m_{Z_{E}}(\theta)\right)$ is defined by:

$$
\begin{aligned}
& -m_{\lambda_{E}}^{-}(\theta) \bar{\lambda}_{E} \leqslant \lambda_{E}(\theta)-\bar{\lambda}_{E} \leqslant m_{\lambda_{E}}^{+}(\theta) \bar{\lambda}_{E} \quad \forall E \in \mathbb{E} \\
& {\left[\bar{e}_{E}\left(\underline{Z}_{E}(\theta)-\underline{Z}_{E}\right)\right]^{\frac{1}{2}} \leqslant m_{Z_{E}}(\theta)^{2}\left[\bar{e}_{E}\left(\bar{Z}_{E}\right)\right]^{\frac{1}{2}} \quad \forall E \in \mathbb{E}}
\end{aligned}
$$

Here, the model of the environment being used is considered to be deterministic. $\underline{F}(\theta)$ characterized by $\left(\lambda_{E}(\theta), \underline{Z}_{E}(\theta)\right)_{E \in \mathbb{E}}$ is the actual applied force distribution, while $\underline{\bar{F}}=\sum\left(\bar{\lambda}_{E} \underline{\bar{Z}}\right)$ is the calculated applied force distribution.

\subsection{Effective LOK of an output of interest}

Let us first introduce the solution $\underline{x}$ of the equation:

$$
\mathbb{K} \underline{x}=\underline{f}
$$

where $\mathbb{K}$ is the stiffness matrix and $f$ is the generalized force. Let $\underline{m}$ be the set of all the basic LOKs associated with the stiffness terms and the applied forces. It is clear that one can associate with each value of $m$ the stiffnesses and forces which verify Eqs. (1), (6) and (7). The corresponding sets are $\mathcal{K}_{m}$ and $\mathcal{F}_{m}$, which are parameterized by $m$.

This approach leads to a new modeling approach for the family of actual structures being studied, in which one defines only the envelope of the actual responses. Precisely, the envelope of the actual responses is associated with Eq. (8), where

$$
\mathbb{K}(\theta) \in \mathcal{K}_{m(\theta)}, \quad \underline{F}(\theta) \in \mathcal{F}_{m(\theta)}
$$

For an output of interest $\alpha(\theta)$ (whose finite element value is $\bar{\alpha}$ ), which can be, for example, a local stress, one can calculate the effective LOK such that

$$
\Delta \alpha^{-}(\theta) \leqslant \alpha(\theta)-\bar{\alpha} \leqslant \Delta \alpha^{+}(\theta)
$$


The output of interest $\alpha$ is a functional of $\mathbb{K}$ and $f$ :

$$
\alpha=\mathbb{L}(\mathbb{K}, \underline{f})
$$

where $\mathbb{L}$ is a given operator. The FE value is:

$$
\bar{\alpha}=\mathbb{L}(\overline{\mathbb{K}}, \underline{\bar{f}})
$$

Consequently, one has:

$$
\begin{aligned}
\Delta \alpha^{+}(\theta)= & \sup _{\substack{\mathbb{K}(\theta) \in \mathcal{K}_{m(\theta)} \\
\underline{F}(\theta) \in \mathcal{F}_{m(\theta)}}}[\mathbb{L}(\mathbb{K}, \underline{f})-\bar{\alpha}] \\
\Delta \alpha^{-}(\theta)= & \inf _{\substack{\mathbb{K}(\theta) \in \mathcal{K}_{m(\theta)} \\
\underline{F}(\theta) \in \mathcal{F}_{m(\theta)}}}[\mathbb{L}(\mathbb{K}, \underline{f})-\bar{\alpha}]
\end{aligned}
$$

If the basic LOKs are small enough, linearization with respect to the deterministic reference model makes the calculation easy. Let us consider:

$$
\alpha=\underline{b}^{T} \underline{x}=\left(\mathbb{K}^{-1} \underline{b}\right)^{T} \underline{f}
$$

One has:

$$
\Delta \alpha=\left(\mathbb{K}^{-1} \underline{b}\right)^{T} \Delta \underline{f}-\underline{f}^{T} \mathbb{K}^{-1}(\Delta \mathbb{K}) \mathbb{K}^{-1} \underline{b}
$$

and

$$
\begin{aligned}
\Delta \alpha^{+}(\theta)= & \sum_{E \in \mathbb{E}}\left[\frac{1}{2}\left(m_{E}^{+}(\theta)+m_{E}^{-}(\theta)\right)\left(\langle\langle\underline{\bar{x}}, \underline{\bar{x}}\rangle\rangle_{E}\right)^{\frac{1}{2}}\left(\left\langle\left\langle\underline{x}_{b}, \underline{x}_{b}\right\rangle\right\rangle_{E}\right)^{\frac{1}{2}}-\frac{1}{2}\left(m_{E}^{+}(\theta)-m_{E}^{-}(\theta)\right)\left\langle\left\langle\underline{\bar{x}}, \underline{x}_{b}\right\rangle\right\rangle_{E}\right. \\
& \left.+m_{\lambda_{E}}^{+}(\theta) \underline{x}_{b_{E}}^{T} \underline{f}_{E}+m_{Z_{E}}(\theta)^{2}\left[\bar{e}_{E}\left(\underline{x}_{b_{E}}\right)\right]^{\frac{1}{2}} \bar{\lambda}_{E}\right] \\
\Delta \alpha^{-}(\theta)= & \sum_{E \in \mathbb{E}}\left[-\frac{1}{2}\left(m_{E}^{+}(\theta)+m_{E}^{-}(\theta)\right)\left(\left\langle\langle\underline{\bar{x}}, \underline{\bar{x}}\rangle_{E}\right)^{\frac{1}{2}}\left(\left\langle\left\langle\underline{x}_{b}, \underline{x}_{b}\right\rangle\right\rangle_{E}\right)^{\frac{1}{2}}-\frac{1}{2}\left(m_{E}^{+}(\theta)-m_{E}^{-}(\theta)\right)\left\langle\left\langle\underline{\bar{x}}, \underline{x}_{b}\right\rangle\right\rangle_{E}\right.\right. \\
& \left.-m_{\lambda_{E}}^{-}(\theta) \underline{x}_{b_{E}}^{T} \underline{f}_{E}-m_{Z_{E}}(\theta)^{2}\left[\bar{e}_{E}\left(\underline{x}_{b_{E}}\right)\right]^{\frac{1}{2}} \bar{\lambda}_{E}\right]
\end{aligned}
$$

Eqs. (16a) and (16b) will be established in Sections 2.2.1 and 2.2.2 respectively.

We have introduced the displacement field $\underline{x}_{b}$ defined by the relation:

$$
\underline{x}_{b}=\overline{\mathbb{K}}^{-1} \underline{b}
$$

Incidentally, $\underline{\bar{x}}$ is the solution of the FE problem with $(\overline{\mathbb{K}}, \bar{f})$. The scalar product $\langle.,$.$\rangle takes into account the actual stiffness:$

$$
\begin{aligned}
& \langle\underline{x}, \underline{y}\rangle=\underline{x}^{T} \mathbb{K} \underline{y} \\
& \langle\underline{x}, \underline{y}\rangle_{E}=\underline{x}^{T} \mathbb{K}_{E} \underline{y}
\end{aligned}
$$

and the second $\langle\langle.,\rangle$.$\rangle includes the deterministic FE stiffness:$

$$
\begin{aligned}
& \langle\langle\underline{x}, \underline{y}\rangle\rangle=\underline{x}^{T} \overline{\mathbb{K}} \underline{y} \\
& \langle\langle\underline{x}, \underline{y}\rangle\rangle_{E}=\underline{x}^{T} \overline{\mathbb{K}}_{E} \underline{y}
\end{aligned}
$$

\subsubsection{Detailed discussion of the propagation of LOKs about stiffness properties}

Let us consider only LOKs relative to stiffness properties. Eq. (15) can be simplified to:

$$
\Delta \alpha=-\underline{f}^{T} \overline{\mathbb{K}}^{-1}(\Delta \mathbb{K}) \overline{\mathbb{K}}^{-1} \underline{b}=-\underline{\bar{x}}^{T}(\Delta \mathbb{K}) \underline{x}_{b}
$$

This expression can be viewed as the difference of two scalar products:

$$
\Delta \alpha=\left\langle\left\langle\underline{\underline{x}}, \underline{x}_{b}\right\rangle\right\rangle-\left\langle\underline{\bar{x}}, \underline{x}_{b}\right\rangle=\sum_{E \in \mathbb{E}}\left\langle\left\langle\underline{\bar{x}}, \underline{x}_{b}\right\rangle\right\rangle_{E}-\left\langle\underline{\bar{x}}, \underline{x}_{b}\right\rangle_{E}
$$

Since the scalar product $\left\langle\underline{\underline{x}}, \underline{x}_{b}\right\rangle_{E}$ is not an energy, Eq. (2) cannot be used directly to bound $\Delta \alpha$. Therefore, one tries to express $\left\langle\underline{\bar{x}}, \underline{x}_{b}\right\rangle_{E}$ as a sum of energies. For example, one has the relation: 


$$
\left\langle\underline{\bar{x}}, \underline{x}_{b}\right\rangle_{E}=\frac{1}{4}\left(\left\langle\underline{\bar{x}}+\underline{x}_{b}, \underline{\bar{x}}+\underline{x}_{b}\right\rangle_{E}-\left\langle\underline{\bar{x}}-\underline{x}_{b}, \underline{\bar{x}}-\underline{x}_{b}\right\rangle_{E}\right)
$$

which, after bounding $\left\langle\underline{\underline{x}}+\underline{x}_{b}, \underline{\bar{x}}+\underline{x}_{b}\right\rangle_{E}$ and $\left\langle\underline{\bar{x}}-\underline{x}_{b}, \underline{\bar{x}}-\underline{x}_{b}\right\rangle_{E}$ using Eq. (2), can lead to the bounds of $\left\langle\underline{\underline{x}}, \underline{x}_{b}\right\rangle_{E}$. In order to obtain the best possible bounds of the quantity of interest, one introduces a scalar $\ell_{E}$ such that:

$$
\left\langle\underline{\bar{x}}, \underline{x}_{b}\right\rangle_{E}=\frac{1}{4 \ell_{E}}\left(\left\langle\underline{\bar{x}}+\ell_{E} \underline{x}_{b}, \underline{\bar{x}}+\ell_{E} \underline{x}_{b}\right\rangle_{E}-\left\langle\underline{\bar{x}}-\ell_{E} \underline{x}_{b}, \underline{\bar{x}}-\ell_{E} \underline{x}_{b}\right\rangle_{E}\right)
$$

Using Eq. (2) again, one obtains the bounds of the two scalar products $\left\langle\underline{\bar{x}}-\ell_{E} \underline{x}_{b}, \underline{\bar{x}}-\ell_{E} \underline{x}_{b}\right\rangle_{E}$ and $\left\langle\underline{\bar{x}}+\ell_{E} \underline{x}_{b}, \underline{\bar{x}}+\ell_{E} \underline{x}_{b}\right\rangle_{E}$. This leads to the following bounds of the scalar product $\left\langle\underline{\underline{x}}, \underline{x}_{b}\right\rangle_{E}$ :

$$
\begin{aligned}
& \left\langle\underline{\bar{x}}, \underline{x}_{b}\right\rangle_{E} \leqslant \frac{1}{4 \ell_{E}}\left[\left(1+m_{E}^{+}(\theta)\right)\left\langle\left\langle\underline{\bar{x}}+\ell_{E} \underline{x}_{b}, \underline{\bar{x}}+\ell_{E} \underline{x}_{b}\right\rangle\right\rangle_{E}-\left(1-m_{E}^{-}(\theta)\right)\left\langle\left\langle\underline{\bar{x}}-\ell_{E} \underline{x}_{b}, \underline{\bar{x}}-\ell_{E} \underline{x}_{b}\right\rangle\right\rangle_{E}\right] \\
& \left\langle\underline{\bar{x}}, \underline{x}_{b}\right\rangle_{E} \geqslant \frac{1}{4 \ell_{E}}\left[\left(1-m_{E}^{-}(\theta)\right)\left\langle\left\langle\underline{\bar{x}}+\ell_{E} \underline{x}_{b}, \underline{\bar{x}}+\ell_{E} \underline{x}_{b}\right\rangle\right\rangle_{E}-\left(1+m_{E}^{+}(\theta)\right)\left\langle\left\langle\underline{\bar{x}}-\ell_{E} \underline{x}_{b}, \underline{\bar{x}}-\ell_{E} \underline{x}_{b}\right\rangle\right\rangle_{E}\right]
\end{aligned}
$$

and finally to the following bounds of the quantity of interest $\Delta \alpha$ :

$$
\Delta \alpha^{-} \leqslant \Delta \alpha \leqslant \Delta \alpha^{+}
$$

with:

$$
\begin{aligned}
& \Delta \alpha^{+}=\sum_{E \in \mathbb{E}}\left(m_{E}^{+}(\theta)+m_{E}^{-}(\theta)\right) \frac{\langle\langle\underline{\underline{x}}, \underline{\underline{x}}\rangle\rangle_{E}+\ell_{E}^{2}\left\langle\left\langle\underline{x}_{b}, \underline{x}_{b}\right\rangle\right\rangle_{E}}{4 \ell_{E}}-\frac{1}{2}\left(m_{E}^{+}(\theta)-m_{E}^{-}(\theta)\right)\left\langle\left\langle\underline{\underline{x}}, \underline{x}_{b}\right\rangle\right\rangle_{E} \\
& \Delta \alpha^{-}=\sum_{E \in \mathbb{E}}-\left(m_{E}^{+}(\theta)+m_{E}^{-}(\theta)\right) \frac{\langle\langle\underline{\underline{x}}, \underline{\underline{x}}\rangle\rangle_{E}+\ell_{E}^{2}\left\langle\left\langle\underline{x}_{b}, \underline{x}_{b}\right\rangle\right\rangle_{E}}{4 \ell_{E}}-\frac{1}{2}\left(m_{E}^{+}(\theta)-m_{E}^{-}(\theta)\right)\left\langle\left\langle\underline{\underline{x}}, \underline{x}_{b}\right\rangle\right\rangle_{E}
\end{aligned}
$$

The set of the optimum scalars $\ell_{E}$ can be obtained by minimizing the gap between $\Delta \alpha^{+}$and $\Delta \alpha^{-}$. This condition is met when:

$$
\ell_{E}=\varepsilon_{E} \sqrt{\frac{\langle\langle\underline{\bar{x}}, \bar{x}\rangle\rangle_{E}}{\left\langle\underline{x_{b}}, \underline{x}_{b}\right\rangle_{E}}} \quad \text { with } \varepsilon_{E}= \pm 1
$$

Then, the gap $\left(\Delta \alpha^{+}-\Delta \alpha^{-}\right)$, which must remain positive, is given by:

$$
\left(\Delta \alpha^{+}-\Delta \alpha^{-}\right)_{O P T}=\sum_{E \in \mathbb{E}} \varepsilon_{E}\left(m_{E}^{+}(\theta)+m_{E}^{-}(\theta)\right)\left(\langle\langle\underline{\underline{x}}, \underline{\bar{x}}\rangle\rangle_{E}\right)^{\frac{1}{2}}\left(\left\langle\left\langle\underline{x}_{b}, \underline{x}_{b}\right\rangle\right\rangle_{E}\right)^{\frac{1}{2}}
$$

In order to ensure that $\Delta \alpha^{+}-\Delta \alpha^{-} \geqslant 0$, one chooses all the $\varepsilon_{E}$ to be positive.

Finally, one gets the following expressions of the optimum bounds $\Delta \alpha^{+}$and $\Delta \alpha^{-}$:

$$
\begin{aligned}
& \Delta \alpha_{O P T}^{+}=\sum_{E \in \mathbb{E}}\left[\frac{1}{2}\left(m_{E}^{+}(\theta)+m_{E}^{-}(\theta)\right)\left(\langle\langle\underline{\bar{x}}, \underline{\bar{x}}\rangle\rangle_{E}\right)^{\frac{1}{2}}\left(\left\langle\left\langle\underline{x}_{b}, \underline{x}_{b}\right\rangle\right\rangle_{E}\right)^{\frac{1}{2}}-\frac{1}{2}\left(m_{E}^{+}(\theta)-m_{E}^{-}(\theta)\right)\left\langle\left\langle\underline{\underline{x}}, \underline{x}_{b}\right\rangle\right\rangle_{E}\right] \\
& \left.\Delta \alpha_{O P T}^{-}=\sum_{E \in \mathbb{E}}\left[-\frac{1}{2}\left(m_{E}^{+}(\theta)+m_{E}^{-}(\theta)\right)\left(\langle\langle\underline{\underline{x}}, \underline{\bar{x}}\rangle\rangle_{E}\right)^{\frac{1}{2}}\left(\left\langle\underline{x}_{b}, \underline{x}_{b}\right\rangle\right\rangle_{E}\right)^{\frac{1}{2}}-\frac{1}{2}\left(m_{E}^{+}(\theta)-m_{E}^{-}(\theta)\right)\left\langle\left\langle\underline{\underline{x}}, \underline{x}_{b}\right\rangle\right\rangle_{E}\right]
\end{aligned}
$$

\subsubsection{Detailed discussion of the propagation of LOKs of the applied forces}

Now, let us consider LOKs about the amplitudes and directions of the applied forces. Eq. (15) can be simplified to:

$$
\Delta \alpha=\left(\mathbb{K}^{-1} \underline{b}\right)^{T} \Delta \underline{f}=\underline{x}_{b}^{T}(\underline{\bar{Z}} \Delta \lambda+\bar{\lambda} \Delta \underline{Z})
$$

Using Eq. (6), the bounds of the amplitude dispersion are obvious: for each substructure $E \in \mathbb{E}$,

$$
-m_{\lambda_{E}}^{-}(\theta) \bar{\lambda}_{E} \underline{x}_{b_{E}}^{T} \underline{\underline{Z}}_{E} \leqslant \underline{x}_{b_{E}}^{T} \underline{\bar{Z}}_{E} \Delta \lambda \leqslant m_{\lambda_{E}}^{+}(\theta) \bar{\lambda}_{E} \underline{x}_{b_{E}}^{T} \underline{\bar{Z}}_{E}
$$

For the propagation of the LOK of the direction $\underline{Z}_{E}$, which is often taken in normalized form such that $\bar{e}_{E}\left(\bar{Z}_{E}\right)=1$,

$$
\left|\underline{x}_{b_{E}}^{T} \bar{\lambda}_{E} \Delta \underline{Z}_{E}\right| \leqslant \bar{\lambda}_{E}\left[e_{E}\left(\underline{x}_{b_{E}}\right)\right]^{\frac{1}{2}}\left[e_{E}(\underline{Z})\right]^{\frac{1}{2}}
$$

Then, using Eq. (7) which defines $m_{Z_{E}}(\theta)$ in the direction $\underline{Z}_{E}$ for the LOK, we obtain the bounds concerning this LOK:

$$
\left|\underline{x}_{b_{E}}^{T} \bar{\lambda}_{E} \Delta \underline{Z}_{E}\right| \leqslant m_{Z_{E}}(\theta)^{2} \bar{\lambda}_{E}\left[e_{E}\left(\underline{x}_{b_{E}}\right)\right]^{\frac{1}{2}}\left[\bar{e}_{E}\left(\bar{Z}_{E}\right)\right]^{\frac{1}{2}}
$$




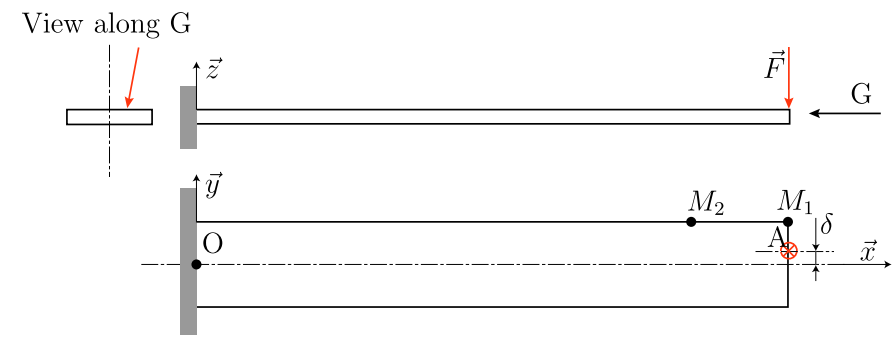

Fig. 1. The structure being studied. The dimensions of the beam are: length $L=435 \mathrm{~mm}$; width $b=50 \mathrm{~mm}$; thickness $h=5.5 \mathrm{~mm}$.

Table 1

Parameters of the Gaussian probabilistic laws describing the variability of $\beta$ and $\delta$.

\begin{tabular}{lll}
\hline Uncertain quantity & Mean & Standard deviation \\
\hline Angle $\beta\left(^{\circ}\right)$ & 0 & 5.0 \\
Offset $\delta(\mathrm{mm})$ & 0 & 4.2 \\
\hline
\end{tabular}

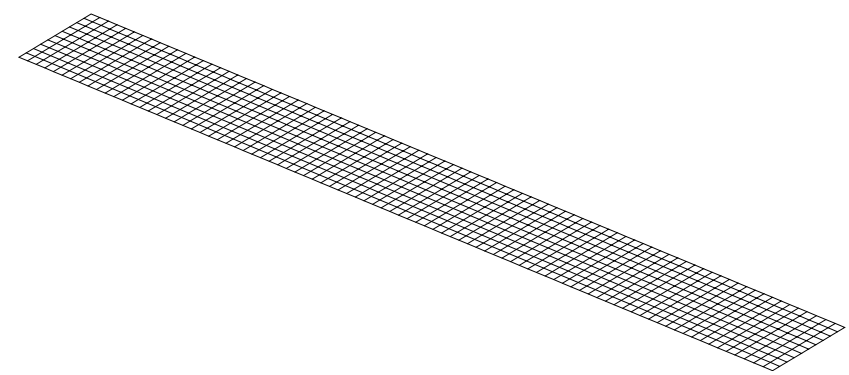

Fig. 2. The mesh used to simulate the experimental data.

which leads to the following expressions for the bounds $\Delta \alpha^{+}$and $\Delta \alpha^{-}$:

$$
\begin{aligned}
& \Delta \alpha^{+}(\theta)=\sum_{E \in \mathbb{E}}\left[m_{\lambda_{E}}^{+}(\theta) \bar{\lambda}_{E} \underline{x}_{b_{E}}^{T} \bar{Z}_{E}+m_{Z_{E}}(\theta)^{2} \bar{\lambda}_{E}\left[e_{E}\left(\underline{x}_{b_{E}}\right)\right]^{\frac{1}{2}}\left[\bar{e}_{E}\left(\bar{Z}_{E}\right)\right]^{\frac{1}{2}}\right] \\
& -\Delta \alpha^{-}(\theta)=\sum_{E \in \mathbb{E}}\left[-m_{\lambda_{E}}^{-}(\theta) \bar{\lambda}_{E} \underline{x}_{b_{E}}^{T} \underline{\bar{Z}}_{E}-m_{Z_{E}}(\theta)^{2} \bar{\lambda}_{E}\left[e_{E}\left(\underline{x}_{b_{E}}\right)\right]^{\frac{1}{2}}\left[\bar{e}_{E}\left(\bar{Z}_{E}\right)\right]^{\frac{1}{2}}\right]
\end{aligned}
$$

\section{Example of propagation and reduction of effective LOKs}

\subsection{Presentation of the example}

The structure being considered is a simple cantilever beam (Fig. 1). An excitation force $\vec{F}$ is applied at the free end with two sources of variability:

- an offset from the $(O, \vec{x})$ axis by a distance denoted $\delta$;

- the angle $\beta$ in the $(A, \vec{y}, \vec{z})$ plane between the excitation $\vec{F}$ and the $(A, \vec{z})$ axis.

\subsection{Simulated experimental data}

Several experiments were simulated using the finite element method and considering that the probability laws associated with the direction and position of the excitation $\vec{F}$ are Gaussian (with the parameters given in Table 1 ). The mesh used consisted of plate elements and is shown in Fig. 2. In order to have the same mesh for all the configurations simulated, the offset $\delta$ of the excitation was not introduced physically, but represented by the same excitation $\vec{F}$, centered, plus an additional torque in the direction $\vec{x}$.

Introducing two quantities of interest, namely the displacements along $\vec{z}$ measured at points $M_{1}$ and $M_{2}$ (see Fig. 1), we used a Monte Carlo simulation to obtain the corresponding probability density functions (Fig. 3).

From the two associated cumulative density functions, we obtained the $99 \%$-probability bounds of the quantities of interest shown in Table 2. The X\%-probability upper bound of a quantity is equal to $Y$ if $X \%$ of the random events lead to 


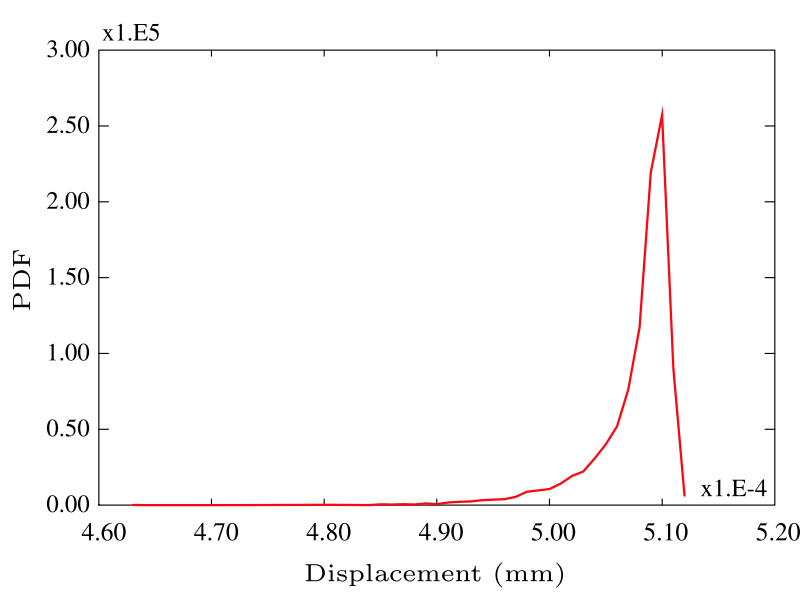

(a) Point $M_{1}$

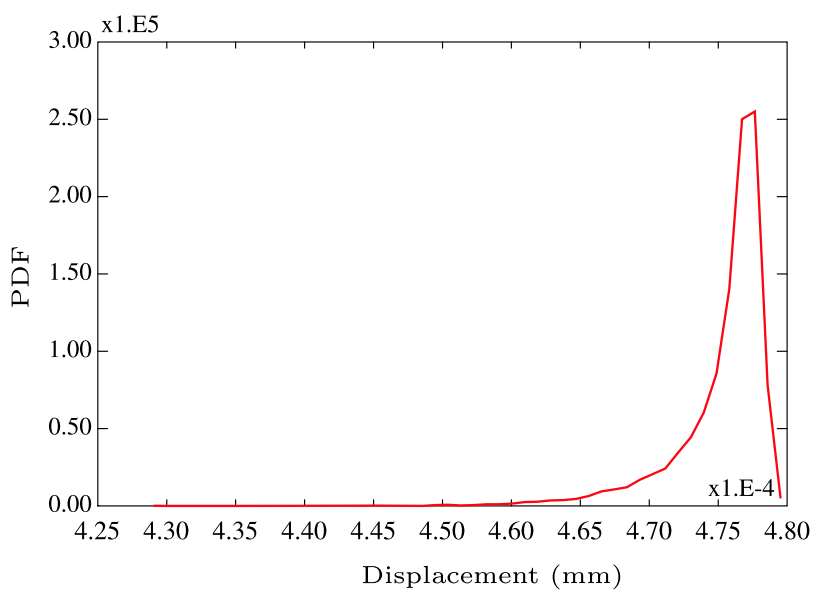

(b) Point $M_{2}$

Fig. 3. The two probability density functions associated with the two quantities of interest obtained from a Monte Carlo simulation of the experimental data.

Table 2

Experimental variability observed for the two quantities of interest (the displacements in direction $\vec{z}$ measured at points $M_{1}$ and $M_{2}$ ) based on a Monte Carlo simulation.

\begin{tabular}{lllll}
\hline Quantity of interest & Point $M_{1}$ & & \multicolumn{2}{l}{ Point $M_{2}$} \\
\cline { 2 - 3 } & $u_{z}^{-}$ & $u_{z}^{+}$ & $u_{z}^{-}$ & $4.54863 \times 10^{-4}$ \\
\hline $99 \%$-probability bounds & $4.91027 \times 10^{-4}$ & $5.11698 \times 10^{-4}$ & $4.74214 \times 10^{-4}$ \\
$\Delta u$ in \% & $-3.8 \%$ & $+0.3 \%$ & $-3.8 \%$ & $+0.3 \%$ \\
\hline
\end{tabular}

a value less than or equal to $Y$. The $X \%$-probability lower bound of a quantity is equal to $Y$ if $X \%$ of the random events lead to a value greater than or equal to $Y$. The absolute displacement $\Delta u$ is the difference between the mean value of the events and the upper or lower 99\%-probability bound associated with a quantity of interest. The relative displacement $\Delta u$ in \% is equal to the absolute displacement $\Delta u$ divided by the mean value of the events.

\subsection{Definition of the basic LOKS}

In order to represent the variabilities in the direction and position of the excitation with basic LOKs, we first introduce the model presented in Fig. 4, in which the excitation is centered on the $(0, \vec{x})$ axis and the experimental offset is described by a torque $M \vec{x}$.

The variabilities of the excitation can be represented using two types of basic LOKs:

- a basic LOK concerning the direction of the excitation $\vec{F}$;

- a pair of basic LOKs concerning the amplitude of the torque $M$.

The deterministic model associated with the basic LOKs represents the case where the offset $\delta$ is equal to zero. In this case, the torque $M$ is also equal to zero, and the introduction of basic LOKs of the amplitude of $M$ is impossible because it would lead to a deterministic response. Instead, we introduce two opposite, nonzero torques along with two associated pairs of identical basic LOKs of the amplitude. The magnitude of these torques is set such that the total torque corresponds to the maximum assumed experimental offset $\delta$, with reasonable basic LOKs of the amplitude (a few \%).

\subsection{Propagation of the basic LOKS}

Table 3 shows the basic LOKs chosen for the model along with the results obtained by propagation of the basic LOKs throughout the finite element model:

- 99\%-probability bounds of the quantities of interest;

- relative $99 \%$-probability bounds (in \%) of the quantities of interest. 


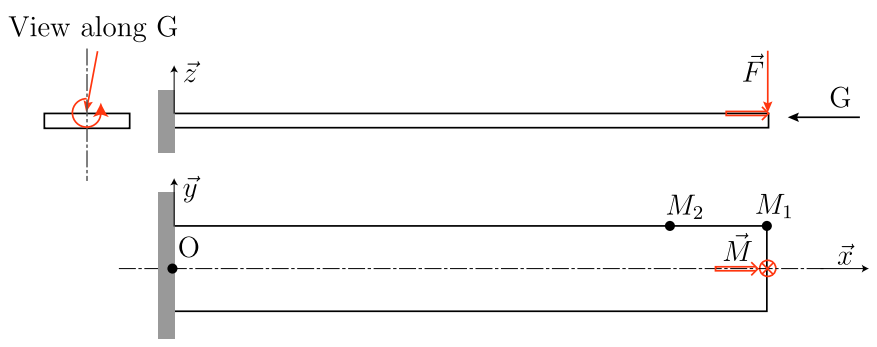

Fig. 4. Equivalent model of the initial problem enabling the introduction of basic LOKs of the excitations.

Table 3

99\%-probability bounds obtained after propagation of the basic LOKs: $\bar{m}_{Z}=0.03$ and $\bar{m}_{\lambda}^{-}=\bar{m}_{\lambda}^{+}=0.03$; the associated error is $e=6.5 \times 10^{-2}$.

\begin{tabular}{llll}
\hline Quantity of interest & Point $M_{1}$ & & \multicolumn{2}{c}{ Point $M_{2}$} \\
\cline { 2 - 4 } & $u_{z}^{-}$ & $u_{z}^{+}$ & $u_{z}^{-}$ \\
\hline Bounds & $4.86413 \times 10^{-4}$ & $5.3470 \times 10^{-4}$ & $4.50692 \times 10^{-4}$ \\
$\Delta u$ in \% & $-4.7 \%$ & $+4.8 \%$ & $4.95427 \times 10^{-4}$ \\
\hline
\end{tabular}

Table 4

99\%-probability bounds obtained after propagation and reduction of the basic LOKs (symmetrical case); the updated LOKs are $\bar{m}_{Z}=0.02$ and $\bar{m}_{\lambda}^{-}=\bar{m}_{\lambda}^{+}=$ 0.029 ; the associated error is $e=5.2 \times 10^{-2}$.

\begin{tabular}{llll}
\hline Quantity of interest & Point $M_{1}$ & & Point $M_{2}$ \\
\hline Bounds & $u_{z}^{-}$ & $u_{z}^{+}$ & $u_{z}^{-}$ \\
Relative bounds & $4.90403 \times 10^{-4}$ & $5.30486 \times 10^{-4}$ & $4.54387 \times 10^{-4}$ \\
$\Delta u$ in \% & $-3.9 \%$ & $+4.0 \%$ & $-3.9 \%$ \\
\hline
\end{tabular}

Table 3 shows that this first LOK model is capable of representing the variability observed during the experiments. However the bounds are too wide compared to the reality observed. In order to improve the quality of the prediction of the LOK model, we now propose to update the initial set of basic LOKs.

\subsection{Updating of the basic LOKs using experimental data}

The approach which one uses to update a deterministic or stochastic finite element model using experimental information can be extended to the modification of the basic LOKs of the model in order to match the envelope of the model's response to that of the experimental response. In order to do that, let us consider the gaps between the lower and upper bounds from both the experiments and the numerical model. Then, the key idea is to seek the basic LOKs which lead to the minimum gap. This idea was already applied to LOKs of structural stiffnesses with quantities of interest such as eigenpulsations $[19,20]$. Here, the gap is calculated, in the least-squares sense, from $99 \%$-probability bounds of the $N_{i}$ quantities of interest:

$$
e^{2}=\sum_{n_{i}=1}^{N_{i}}\left(\alpha_{n_{i}, \bmod }^{+}-\alpha_{n_{i}, \text { exp }}^{+}\right)^{2}+\left(\alpha_{n_{i}, \bmod }^{-}-\alpha_{n_{i}, \exp }^{-}\right)^{2}
$$

First, the basic LOKs $\bar{m}_{\lambda}^{+}$and $\bar{m}_{\lambda}^{-}$are set to the same values. The basic LOKs which minimize the error introduced in (37) are given in Table 4. This table shows that the lower bound obtained with the LOK model is very close to the experimental lower bound, but after this first updating the quality of the upper bound could not be improved. In fact, as shown in Eqs. (36a) and (36b), the bounds obtained for each quantity of interest are globally symmetrical in the case of identical basic LOKs. Since the experimental results are nonsymmetrical (see Table 2) this first model cannot be used to represent reality accurately.

Therefore, we now propose nonsymmetrical basic LOKs of the amplitudes of the torques. Fig. 5 shows the evolution of the error $e$ as a function of the two nonsymmetrical basic LOKs $\bar{m}_{\lambda}^{-}$and $\bar{m}_{\lambda}^{+}$. The results are improved by choosing the optimum values for $\bar{m}_{\lambda}^{-}$and $\bar{m}_{\lambda}^{+}$. The envelope of the LOK model matches the experimental bounds very well (see Table 5 ).

This example shows the interest of the LOK modeling approach in order to take into account uncertainties concerning applied forces: with the introduction of a very small number of internal variables through two LOKs for the amplitude and one LOK for the direction of the excitation, we can guarantee that the finite element LOK model gives the best possible representation of reality. Remarkably, this model does not require any assumption concerning the stochastic representation of the uncertain forces in order to predict the structural response envelopes. 
Table 5

99\%-probability bounds obtained after propagation and reduction of the basic LOKs (nonsymmetrical case); the updated LOKs are $\bar{m}_{Z}=0.0135, \bar{m}_{\lambda}^{-}=0.025$ and $\bar{m}_{\lambda}^{+}=0.0020$; the associated error is $e=5.6 \times 10^{-3}$.

\begin{tabular}{llll}
\hline Quantity of interest & Point $M_{1}$ & & Point $M_{2}$ \\
\hline & $u_{z}^{-}$ & $u_{z}^{+}$ & $u_{z}^{-}$ \\
Bounds & $4.89089 \times 10^{-4}$ & $5.12253 \times 10^{-4}$ & $4.53169 \times 10^{-4}$ \\
$\Delta u$ in \% & $-4.1 \%$ & $+0.4 \%$ & $4.74627 \times 10^{-4}$ \\
\hline
\end{tabular}

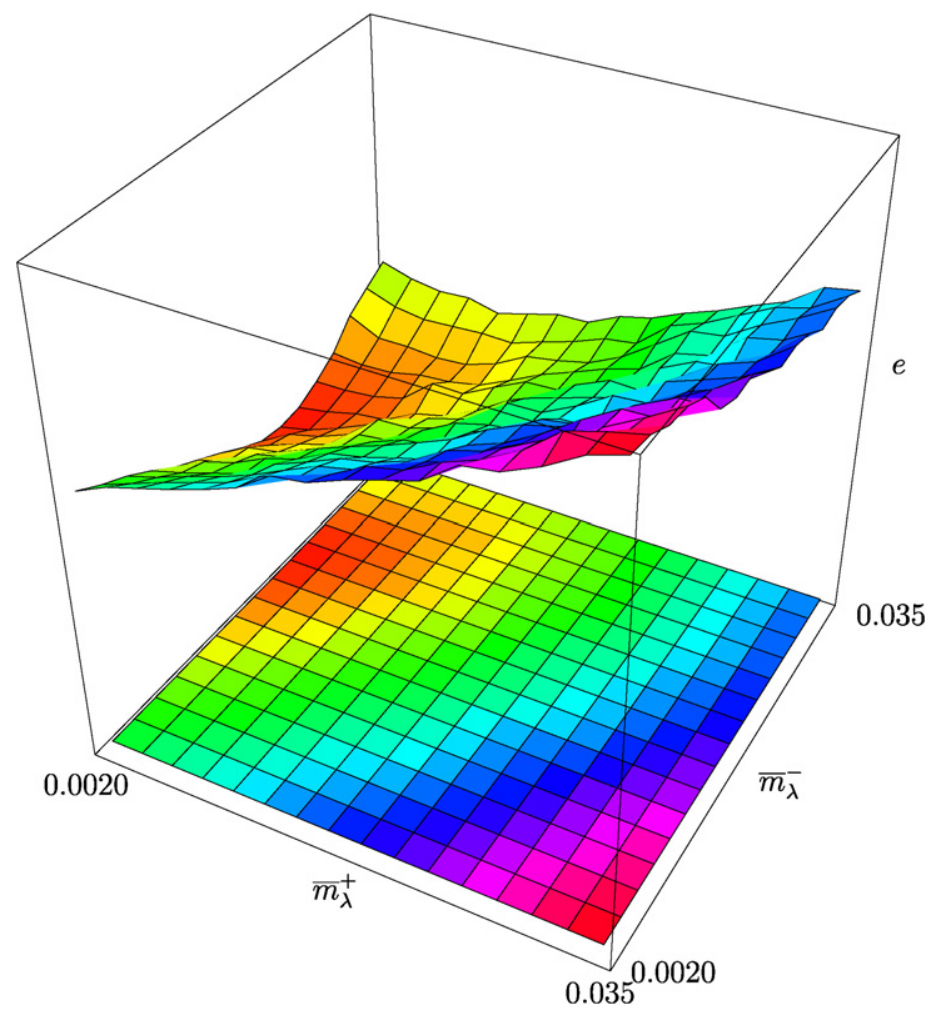

Fig. 5. Error $e$ as a function of the two basic LOKs $\bar{m}_{\lambda}^{-}$and $\bar{m}_{\lambda}^{+}$, with $\bar{m}_{Z}=0.0135$.

\section{Conclusion}

In this article, we carried out a detailed analysis of the LOK theory as a solution to the validation problem. This original approach consists in inserting internal variables into the model in the form of basic LOKs, then predicting the structural response envelopes which contain the real responses. We presented an example which illustrates how this approach works and what its capabilities are.

Experimental results can be taken into account in order to improve a first LOK model. In order to do that, one introduces a distance between the envelopes of the experiments and the LOK model, and the minimization of this distance leads to the optimum set of basic LOKs.

Then, the validation is associated with the width of the envelope.

\section{References}

[1] K. Popper, The Logic of Scientific Discovery, Routledge Classics, Taylor and Francis, 2003.

[2] J.T. Oden, T. Belytschko, I. Babuska, T. Hughes, Research directions in computational mechanics, Computer Methods in Applied Mechanics and Engineering 192 (2003) 913-922.

[3] F.M. Hemez, S.W. Doebling, M.C. Anderson, A brief tutorial on verification and validation, in: Proceedings of the 22nd International Modal Analysis Conference (IMAC-XXII), Dearborn, Michigan, January 26-29, 2004.

[4] W. Oberkampf, T. Trucano, C. Hirsh, Verification, validation and predictive capability in computational engineering and physics, Technical report, Sandia Report 2003-3769, 2003.

[5] A. Saltelli, S. Tarantola, F. Campolongo, M. Ratto, Sensitivity Analysis in Practice: A Guide to Assessing Scientific Models, John Wiley and Sons, 2004.

[6] H. Berger, R. Ohayon, L. Quetin, L. Barthe, P. Ladevèze, M. Reynier, Updating methods for structural dynamics models, La Recherche Aérospatiale 5 (1991) 9-20 (in French).

[7] C. Farhat, F. Hemez, Updating finite element dynamics models using an element-by-element sensitivity methodology, AIAA Journal 31 (9) (1993) $1702-1711$. 
[8] J. Piranda, G. Lallement, S. Cogan, Parametric correction of finite element modes by minimization of an output residual: improvement of the sensitivity method, in: Proc. IMAC IX, Firenze, Italy, 1991, pp. 363-368.

[9] S. Lammens, M. Brughmans, J. Leuridan, W. Heylen, P. Sas, Application of a FRF based model updating technique for the validation of a finite element model of components of the automotive industry, in: ASME Conference, Boston, 1995, pp. 1191-1200.

[10] P. Ladevèze, M. Reynier, FE modeling and analysis: a localization method of stiffness errors and adjustments of FE models, in: Vibrations Analysis Techniques and Application, ASME Publishers, 1989, pp. 355-361.

[11] B. Faverjon, P. Ladevèze, F. Louf, Validation of stochastic linear structural dynamics models, Computers and Structures 87 (13-14) (2009) 829-837.

[12] P. Ladevèze, On a theory of the lack of knowledge in structural computation, Technical Note SY/XS 136 127, EADS Launch Vehicles, April 2002 (in French).

[13] P. Ladevèze, Model validation or how can one describe the lack of knowledge, IACM Expressions, 2005.

[14] G.I. Schuëller, Computational stochastic mechanics - recent advances, Computers and Structures 79 (2001) 2225-2234.

[15] Y. Ben-Haim, Information-Gap Decision Theory, Academic Press, London, 2001.

[16] G. Klir, Generalized information theory, Fuzzy Sets and Systems 40 (1991) 127-142.

[17] P. Walley, Statistical Reasoning with Imprecise Probabilities, Chapman and Hall, 1990.

[18] R.M. Moore, Methods and Applications of Interval Analysis, Studies in Applied Mathematics (SIAM), 1979.

[19] P. Ladevèze, G. Puel, T. Romeuf, Lack of knowledge in structural model validation, Computer Methods in Applied Mechanics and Engineering 195 (July 2006) 4697-4710.

[20] P. Ladevèze, G. Puel, T. Romeuf, Reduction of the lack of knowledge of an industrial structural dynamics model, in: 8th US National Congress on Computational Mechanics, Austin, Texas, July 25-27, 2005. 